\title{
Herniae: benign or malignant?
}

\author{
I. R. HAYWOOD \\ F.R.C.S.
}

British Military Hospital, Münster, BFPO 17

\section{Summary}

Three cases of cancer presenting at inguinal hernia repair are described. The importance of the awareness of this possibility is emphasized, and one finger routine mini-laparotomy exploration is recommended. In suspicious cases further access via epigastric vessel division is also recommended as is the value of histological examination of the hernial sac.

\section{Introduction}

Inguinal herniotomy is perhaps the most commonly performed elective surgical procedure, either alone or as the first stage of a herniorrhaphy. During this procedure the peritoneal cavity is breached, and this may give an opportunity to the surgeon to diagnose a concurrent intra-abdominal pathology. The following three cases were taken to the operating theatre for routine herniorrhaphy by the author within a twelve week period.

\section{Case reports}

\section{Case 1}

A 65-year-old male was admitted with a 4 month history of right inguinal hernia associated with colicky abdominal pain. Intermittently, there had been some pain in his hernia on defaecation and the stools had been somewhat looser than normal since the development of his hernia. A standard preoperative clinical examination showed no other abnormality.

At operation a small indirect hernia sac was found with a marked bulge in the posterior wall of the inguinal canal. On opening the sac a moderate amount of blood-stained ascitic fluid was released from the peritoneal cavity. The epigastric vessels were divided to facilitate examination of the peritoneal cavity. A malignant mass could then be palpated arising from the pelvis. After repairing the hernia, the rectum was examined in the lithotomy position whilst the patient was still under the general anaesthetic. Digital examination was normal, but by means of the bimanual technique the presence of a mass could be confirmed and a sigmoidoscopy showed a rectal carcinoma $13 \mathrm{~cm}$ from the anal verge. Palliative anterior resection for a well differentiated capillary adenocarcinoma was performed three weeks later.

\section{Case 2}

A 75-year-old man was admitted with acute retention of urine. There had been nocturia for 4 weeks before admission, but no other urinary trac $B$ symptoms. He had noted vague pains in the left iliae fossa for some months and a dragging pain in a long standing left inguinal hernia, the onset of which had coincided with that of his nocturia.

On examination, the abdomen was found to be grossly obese and a note was made that there might possibly be an ill-defined central abdominal mass. There was a large easily reducible left inguinal hernia. His acute urinary retention was relieved by urinary catheterization and it was found on removing the catheter not to have recurred. Repair of his hernia was therefore elected.

At operation he was found to have an en glissade hernia, the fundus of the bladder forming the medial wall of a tense sac which passed to the bottom of the scrotum. The sac contained a moderate quantity of slightly purulent ascitic fluid. The serosal lining of the sac and coverings of the bladder were inflamed, and the bladder itself was enlarged and its wall thickened. The inferior epigastric vessels were divided after ligation to allow better examination of the abdominal contents. After this manoeuvre it was possible to feel a mass within the pelvic colon. The hernia was repaired and an orchidectomy was performed to facilitate the procedure. Histological examination of the hernia sac showed it to be heavily infiltrated with moderately well differentiated adenocarcinoma, with cellular features suggestive of gastric rather than colonic origin. Subsequently a barium meal showed no evidence of gastric carcinoma. A 
barium enema was reported as showing a long segment of diverticular disease in the pelvic colon, but this had no features suggestive of malignancy. The patient declined any further investigation or surgical exploration and was therefore treated with a course of palliative chemotherapy for malignant ascites of unproven origin.

\section{Case 3}

An 80-year-old man was admitted with a 5 week history of increasing pain and irreducibility of his long-standing right inguinal hernia. On examination he had a large irreducible right inguinal hernia, but there were no signs of intestinal obstruction. The hernia was therefore explored electively.

At operation all the tissues were found to be thickened, including the wall of the sac. Within the sac was a loop of small bowel with what appeared to be an oedematous thickened wall. The opinion formed at the time of operation was that this was most likely to represent a loop of bowel thickened as a result of a recent period of incarceration within the sac. However in view of this unusual appearance, the affected segment of small bowel, which measured about $9 \mathrm{~cm}$, was resected and the hernia repaired in the standard fashion. Histology showed the bowel to be the site of a diffuse, poorly differentiated, malignant lymphoma.

\section{Discussion}

The diagnosis of unexpected disease during hernia repair is well known. Geelhoed, Millar and Ketchan (1974) describe seven cases of malignancy presenting to the National Cancer Institute which were discovered in this manner, and also reviewed the previous publications of other groin masses mis-diagnosed as hernia. Many of these were benign and included lesions of the round ligament in the female, infarction in lymph nodes, torsion of a cryptorchid, thrombophlebitis of a saphena varix, haematomata within the retroperitoneal tissues and spermatic cord, supernumerary bursae around the pectineus, and various benign neoplasms. In all cases the misdiagnosis had been the well-known diagnostic pitfall of an irreducible inguinal hernia. Often this can be avoided by careful history and thorough physical examination, although it must be admitted that in many cases surgical exploration is the means of making the diagnosis.

The discovery of pathology within the sac of a reducible hernia is not uncommon. Acutely inflamed appendices are not infrequent and an inflamed Meckel's diverticulum (Littre's hernia) is a rare finding. Intraperitoneal suppuration presenting as collections of pus within the hernia sac was described by Cronin and Ellis (1959), but these all required emergency exploration. Examination of the fluid contents of a hernial sac is not a commonly used diagnostic procedure but has been described and advocated by Cardan (1975) to confirm the presence of haemoperitoneum should the patient coincidentally have an inguinal hernia. He considered this a better procedure than previously described abdominal paracentesis.

The presence of an intra-abdominal neoplastic tumour prolapsing into a hernia sac does not appear to be well described. Elliott (1972) reports the case of an epidermoid carcinoma of the bladder prolapsing in such circumstances, but a pre-operative diagnosis had been made in this case. However, Oppenheimer (1943) reported coincidental finding of transitional cell bladder carcinoma during hernia repair, and Marcus (1953) one in which three small transitional cell papillomata were found during partial cystectomy performed during hernia repair. Unsuspected bowel malignancy presenting as in the present cases has not been previously described, although Geelhoed, Millar and Ketchen (1974) comment that the sudden development of a hernia should give rise to the suspicion that this might be a process secondary to the development of an intra-abdominal neoplasm. Cases 1 and 2 demonstrate this point. The development of sudden pain and symptoms in a long standing hernia as in Case 3 should also be regarded with suspicion (Ficarra, 1971).

Inguinal herniae and early undiagnosed intraabdominal malignancy are both common conditions, so their coincidental occurrence cannot be rare. The occurrence of these three cases described presenting to the same surgeon within a short period of time can be considered no more than coincidence, but serves to emphasize the point that at herniotomy there is the chance to diagnose unsuspected intra-abdominal neoplasia. Inguinal herniae are often repaired by the most junior members of the surgical team and there is little in the text books or recent publications to emphasize this point. Suspicion of further pathology should certainly be aroused by the presence of anything other than the smallest quantity of fluid within the sac (although of course this may be due to intermittent incarceration of the hernia). Should there be sufficient suspicion a wider examination of the abdominal cavity can be carried out simply by the manoeuvre described, that of dividing the inferior epigastric vessels, and the value of histological examination of the sac removed is also shown. Since encountering the above described cases the author has made it his routine practice in all but young patients to carry out a one finger digital examination of as much of the abdominal contents as possible when a herniotomy is performed and the neck of the sac permits. Perhaps this should be taught as part of the definitive repair of an inguinal hernia. 


\section{Acknowledgments}

I am grateful to Mr J. Cook and Mr J. Ross for permission to publish details of patients under their care, and to Professor G. W. Taylor for his advice.

\section{References}

CARDAN, E. (1975) Letter. Archives of Surgery, 110, 128.

Cronin, K. \& Ellis, H. (1959) Pus collection in hernia sacs, an unusual complication of peritonitis. British Journal of Surgery, 46, 364.
ELLIOTT, R.G. (1972) Incarcerated hernia of bladder containing an epidermoid carcinoma. Southern Medical Journal, 65, 1019.

FICARRA, B.J. (1971) Hernia-masquerades of surgical disorders." Surgical Clinics of North America, 51, 1401.

GeelHOEd, G.W., MillaR, R.C. \& KeTChan, A.S. (1974) Hernia presentations of cancer in the groin. Surgery, 75, 436.

MARCUS, R. (1953) Scrotal cystoceles: report of a case with papillomata. British Journal of Surgery, 41, 182.

OPPENHEIMER, G.D. (1943) Incarcerated inguinal hernia containing carcinoma of bladder. Journal of Urology, 50, 784. 